\title{
HERMITE PSEUDOSPECTRAL METHOD FOR NONLINEAR PARTIAL DIFFERENTIAL EQUATIONS
}

\author{
Ben-Yu GuO ${ }^{1}$ AND Cheng-LONG $\mathrm{XU}^{2}$
}

\begin{abstract}
Hermite polynomial interpolation is investigated. Some approximation results are obtained. As an example, the Burgers equation on the whole line is considered. The stability and the convergence of proposed Hermite pseudospectral scheme are proved strictly. Numerical results are presented.
\end{abstract}

Mathematics Subject Classification. 65M70, 65N30, 76D99.

Received: August 23, 1999. Revised: February 29, 2000.

\section{INTRODUCTION}

Many problems in science and engineering are set in unbounded domains. We may solve them by restricting calculations to some bounded domains with artificial boundary conditions. Whereas this treatment causes errors. A reasonable way is to approximate them by certain orthogonal systems in unbounded domains. Maday et al. [13], Coulaud et al. [3], and Funaro [5] used the Laguerre spectral method for some linear problems. Funaro and Kavian [6] considered some algorithms by using Hermite functions. Recently Guo [10] developed the spectral method by using Hermite polynomials. However it is not easy to perform the quadratures in unbounded domains, which are used in the Hermite spectral approximations. So the Hermite pseudospectral method is more preferable in actual calculations. But so far, there is no work concerning it. The aim of this paper is to develop the Hermite pseudospectral method. We establish some approximation results in the next section. Then as an example, we provide a Hermite pseudospectral scheme for the Burgers equation on the whole line, and prove its stability and the spectral accuracy in Section 3. The numerical results are presented in the final section, which show the high accuracy and the convergence of this method.

\section{HeRMite INTERPOLATION IN ONE DIMENSION}

Let $\Lambda=\{x \mid-\infty<x<\infty\}$ and $\omega(x)=\mathrm{e}^{-x^{2}}$. For $1 \leq p \leq \infty$, let

$$
L_{\omega}^{p}(\Lambda)=\left\{v \mid v \text { is measurable and }\|v\|_{L_{\omega}^{p}}<\infty\right\}
$$

Keywords and phrases. Hermite pseudospectral approximation, nonlinear partial differential equations.

${ }^{1}$ Department of Mathematics, Shanghai Normal University, Shanghai 200234, P.R. China. e-mail: byguo@guomai.sh.cn

2 Department of Mathematics, Shanghai University, Shanghai 201800, P.R. China. 
where

$$
\|v\|_{L_{\omega}^{p}}= \begin{cases}\left(\int_{\Lambda}|v(x)|^{p} \omega(x) \mathrm{d} x\right)^{\frac{1}{p}}, & 1 \leq p<\infty, \\ \underset{x \in \Lambda}{\operatorname{ess} \sup _{x \in \Lambda}|v(x)|,} & p=\infty .\end{cases}
$$

In particular, $L_{\omega}^{2}(\Lambda)$ is a Hilbert space with the inner product

$$
(u, v)_{L_{\omega}^{2}(\Lambda)}=\int_{\Lambda} u(x) v(x) \omega(x) \mathrm{d} x .
$$

Let $\partial_{x} v(x)=\frac{\partial v}{\partial x}(x)$, etc. For any non-negative integer $m$,

$$
H_{\omega}^{m}(\Lambda)=\left\{v \mid \partial_{x}^{k} v \in L_{\omega}^{2}(\Lambda), 0 \leq k \leq m\right\}
$$

equipped with the following inner product, semi-norm and the norm

$$
\begin{gathered}
(u, v)_{H_{\omega}^{m}(\Lambda)}=\sum_{k=0}^{m}\left(\partial_{x}^{k} u, \partial_{x}^{k} v\right)_{\omega}, \\
|v|_{H_{\omega}^{m}(\Lambda)}=\left\|\partial_{x}^{m} v\right\|_{L_{\omega}^{2}(\Lambda)}, \quad\|v\|_{H_{\omega}^{m}(\Lambda)}=(v, v)_{H_{\omega}^{\frac{1}{2}}(\Lambda)} .
\end{gathered}
$$

For any real $r>0$, the space $H_{\omega}^{r}(\Lambda)$ is defined by the space interpolation as in [1]. For simplicity, denote the seminorm $|v|_{H_{\omega}^{r}(\Lambda)}$ and the norm $\|v\|_{H_{\omega}^{r}(\Lambda)}$ by $|v|_{r, \omega}$ and $\|v\|_{r, \omega}$, respectively. In particular, $(u, v)_{\omega}=(u, v)_{L_{\omega}^{2}(\Lambda)}$, $\|v\|_{\omega}=\|v\|_{0, \omega}$ and $\|v\|_{\infty}=\|v\|_{L^{\infty}(\Lambda)}$. Throughout this paper, we denote by $c$ a generic positive constant independent of any function. Guo [10] proved that for any $v \in H_{\omega}^{1}(\Lambda)$,

$$
\begin{aligned}
|v(x)| & \leq 2 e^{\frac{x^{2}}{2}}\|v\|_{\omega}^{\frac{1}{2}}\|v\|_{1, \omega}^{\frac{1}{2}}, \\
\|x v\|_{\omega} & \leq\|v\|_{1, \omega} .
\end{aligned}
$$

The Hermite polynomial of degree $l$ is defined by

$$
H_{l}(x)=(-1)^{l} \mathrm{e}^{x^{2}} \partial_{x}^{l}\left(\mathrm{e}^{-x^{2}}\right) .
$$

Clearly $H_{0}(x) \equiv 1$ and $H_{1}(x)=2 x$. We have

$$
\partial_{x} H_{l}(x)=2 l H_{l-1}(x), \quad l \geq 1 .
$$

The set of Hermite polynomials is the $L_{\omega}^{2}(\Lambda)$-orthogonal system, i.e.,

$$
\int_{\Lambda} H_{l}(x) H_{m}(x) \omega(x) \mathrm{d} x=\gamma_{l} \delta_{l, m}
$$

where $\delta_{l, m}$ is the Kronecker function, and $\gamma_{l}=2^{l} l ! \sqrt{\pi}$.

We first recall some properties of the Hermite approximation. Let $N$ be any positive integer and $\mathcal{P}_{\mathcal{N}}$ be the set of all algebraic polynomials of degree at most $N$. The $L_{\omega}^{2}(\Lambda)$-orthogonal projection $P_{N}: L_{\omega}^{2}(\Lambda) \rightarrow \mathcal{P}_{\mathcal{N}}$ is such a mapping that for any $v \in L_{\omega}^{2}(\Lambda)$,

$$
\left(v-P_{N} v, \phi\right)_{\omega}=0, \quad \forall \phi \in \mathcal{P}_{\mathcal{N}} .
$$

As pointed out in [9], $P_{N}$ is also the best approximations associated with the inner product of the space $H_{\omega}^{m}(\Lambda)$, $m$ being any non negative integer. 
Lemma 2.1 (See [9]). For any $v \in H_{\omega}^{r}(\Lambda)$ and $0 \leq \mu \leq r$,

$$
\left\|v-P_{N} v\right\|_{\mu, \omega} \leq c N^{\frac{\mu-r}{2}}\|v\|_{r, \omega} .
$$

Lemma 2.2. For any $v \in H_{\omega}^{1}(\Lambda)$ and $r \geq 1$,

$$
\left\|\left(v-P_{N} v\right) \mathrm{e}^{-\frac{x^{2}}{2}}\right\|_{\infty} \leq c N^{\frac{1}{4}-\frac{r}{2}}\|v\|_{1, \omega} .
$$

Proof. By (2.1) and Lemma 2.1, for any $x \in \Lambda$,

$$
\begin{aligned}
\left|\left(v-P_{N} v\right) \mathrm{e}^{-\frac{x^{2}}{2}}\right| & \leq 2\left\|v-P_{N} v\right\|_{\omega}^{\frac{1}{2}}\left\|v-P_{N} v\right\|_{1, \omega}^{\frac{1}{2}} \\
& \leq c N^{\frac{1}{4}-\frac{r}{2}}\|v\|_{1, \omega}
\end{aligned}
$$

We now turn to the Hermite-Gauss interpolation. We first introduce some notations. For any two sequences of $\left\{a_{l}\right\}$ and $\left\{b_{l}\right\}$ of nonzero real numbers, we write $a_{l} \preceq b_{l}$, if there exists a positive constant $d$ independent of $l$, such that $a_{l} \leq d b_{l}$ for all $l$ large enough. Moreover we write $a_{l} \sim b_{l}$, if $a_{l} \preceq b_{l}$ and $b_{l} \preceq a_{l}$. Let $\sigma_{j}(0 \leq j \leq N)$ be the $N+1$ simple zeros of $H_{N+1}(x), \sigma_{N}<\sigma_{N-1}<\ldots<\sigma_{0}$. They are situated symmetrically around the origin, see [17]. Let $\Delta_{j}=\sigma_{j-1}-\sigma_{j+1}$ and $a_{N}=\sqrt{2 N}$ be the $N$ th Mhaskar-Rahmanov-Saff number. It is proved in [11] that

$$
-a_{N+1}\left(1-N^{-\frac{2}{3}}\right) \preceq \sigma_{N}, \quad \sigma_{0} \preceq a_{N+1}\left(1-N^{-\frac{2}{3}}\right),
$$

and uniformly for $N \geq 1$ and $1 \leq j \leq N-1$,

$$
\Delta_{j} \sim \frac{1}{\sqrt{N+1}}\left(1-\frac{\left|\sigma_{j}\right|}{a_{N+1}}\right)^{-\frac{1}{2}} .
$$

Let $\Lambda_{N}=\left\{\sigma_{j} \mid 0 \leq j \leq N\right\}$. For any $v \in C(\Lambda)$, the Hermite-Gauss interpolant $I_{N} v \in \mathcal{P}_{N}$ is determined by

$$
I_{N} v(x)=v(x), \quad x \in \Lambda_{N}
$$

Next, Let $\omega_{j}$ be the Christoffel numbers with respect to $\omega(x)$,

$$
\omega_{j}=\frac{2^{N} N ! \sqrt{\pi}}{(N+1) H_{N}^{2}\left(\sigma_{j}\right)}, \quad 0 \leq j \leq N
$$

It is shown as in Lubinsky and Moricz [12] that for $0 \leq j \leq N$,

$$
\left|\partial_{x} H_{N+1}\left(\sigma_{j}\right)\right| \sim 2^{\frac{N}{2}} N^{\frac{1}{4}} \sqrt{(N+1) !} \pi^{\frac{1}{4}} \mathrm{e}^{\frac{\sigma_{j}^{2}}{2}}\left(1-\frac{\left|\sigma_{j}\right|}{a_{N+1}}\right)^{\frac{1}{4}} .
$$

Thus by (2.3),

and so

$$
\left|H_{N}\left(\sigma_{j}\right)\right|^{2} \sim 2^{N} N^{-\frac{3}{2}}(N+1) ! \pi^{\frac{1}{2}} \mathrm{e}^{\sigma_{j}^{2}}\left(1-\frac{\left|\sigma_{j}\right|}{a_{N+1}}\right)^{\frac{1}{2}}
$$

$$
\omega_{j} \sim \frac{1}{\sqrt{N}} \mathrm{e}^{-\sigma_{j}^{2}}\left(1-\frac{\left|\sigma_{j}\right|}{a_{N+1}}\right)^{-\frac{1}{2}}
$$


The discrete inner product and norm related to the Hermite-Gauss interpolation are as follows,

$$
(u, v)_{\omega, N}=\sum_{j=0}^{N} u\left(\sigma_{j}\right) v\left(\sigma_{j}\right) \omega_{j}, \quad\|v\|_{\omega, N}=(v, v)_{\omega, N}^{\frac{1}{2}} .
$$

Clearly

$$
\left(I_{N} v-v, \phi\right)_{\omega, N}=0, \quad \forall \phi \in \mathcal{P}_{N} .
$$

In order to study the properties of the Hermite-Gauss interpolation, we need some preparations. Firstly, by Szegö [16], for any $\phi \in \mathcal{P}_{2 N+1}$,

$$
\int_{\Lambda} \phi(x) \omega(x) \mathrm{d} x=\sum_{j=0}^{N} \phi\left(\sigma_{j}\right) \omega_{j} .
$$

By (2.9), we assert that for any $\phi \psi \in \mathcal{P}_{2 N+1}$,

$$
(\phi, \psi)_{\omega}=(\phi, \psi)_{\omega, N},
$$

and for any $\phi \in \mathcal{P}_{N}$,

$$
\|\phi\|_{\omega}=\|\phi\|_{\omega, N} .
$$

Furthermore let

$$
I_{N} v(x)=\sum_{l=0}^{N} \tilde{v}_{l} H_{l}(x)
$$

By $(2.8)$, for any $v \in C(\Lambda)$,

$$
\left(v, H_{l}\right)_{\omega, N}=\left(I_{N} v, H_{l}\right)_{\omega, N}=\left(I_{N} v, H_{l}\right)_{\omega}=\gamma_{l} \tilde{v}_{l}, \quad 0 \leq l \leq N .
$$

Hence we can take (2.8) as the definition of $I_{N}$.

We are going to the main result in this section.

Lemma 2.3. For any $v \in H_{\omega}^{1}(\Lambda)$,

$$
\|v\|_{\omega, N} \leq c N^{\frac{1}{3}}\|v\|_{\omega}+c N^{-\frac{1}{6}}\|v\|_{1, \omega} .
$$

Proof. By virtue of (2.7),

$$
\|v\|_{\omega, N}^{2} \leq c_{1} N^{-\frac{1}{2}} \sum_{j=0}^{N} \mathrm{e}^{-\sigma_{j}^{2}} v^{2}\left(\sigma_{j}\right)\left(1-\frac{\left|\sigma_{j}\right|}{a_{N+1}}\right)^{-\frac{1}{2}} .
$$

It is shown in [2] that for $-\infty<a<b<\infty$,

$$
\sup _{x \in[a, b]}|v(x)|^{2} \leq \frac{c_{2}}{b-a}\|v\|_{L^{2}(a, b)}^{2}+c_{2}(b-a)|v|_{H^{1}(a, b)}^{2} .
$$

Let $\Lambda_{j}=\left(\sigma_{j+1}, \sigma_{j-1}\right), 1 \leq j \leq N$. Then

$$
\mathrm{e}^{-\sigma_{j}^{2}} v^{2}\left(\sigma_{j}\right) \leq \frac{c_{2}}{\Delta_{j}} \int_{\Lambda_{j}} \mathrm{e}^{-x^{2}} v^{2}(x) \mathrm{d} x+c_{2} \Delta_{j} \int_{\Lambda_{j}}\left(\partial_{x}\left(\mathrm{e}^{-\frac{x^{2}}{2}} v(x)\right)\right)^{2} \mathrm{~d} x .
$$


Moreover the combination of (2.1) and (2.5) leads to that for $j=0, N$,

$$
\mathrm{e}^{-\sigma_{j}^{2}} v^{2}\left(\sigma_{j}\right)\left(1-\frac{\left|\sigma_{j}\right|}{a_{N+1}}\right)^{-\frac{1}{2}} \leq c_{3} N^{\frac{1}{3}}\|v\|_{\omega}\|v\|_{1, \omega} .
$$

By substituting (2.13) and (2.14) into (2.12), we assert that

$$
\begin{aligned}
\|v\|_{\omega, N}^{2} \leq & c_{1} c_{3} N^{-\frac{1}{6}}\|v\|_{\omega}\|v\|_{1, \omega}+c_{1} c_{2} N^{-\frac{1}{2}} \sum_{j=1}^{N-1} \Delta_{j}^{-1}\left(1-\frac{\left|\sigma_{j}\right|}{a_{N+1}}\right)^{-\frac{1}{2}} \int_{\Lambda_{j}} \mathrm{e}^{-x^{2}} v^{2}(x) \mathrm{d} x \\
& +c_{1} c_{2} N^{-\frac{1}{2}} \sum_{j=1}^{N-1} \Delta_{j}\left(1-\frac{\left|\sigma_{j}\right|}{a_{N+1}}\right)^{-\frac{1}{2}} \int_{\Lambda_{j}} \mathrm{e}^{-x^{2}}\left(x^{2} v^{2}(x)+\left(\partial_{x} v(x)\right)^{2}\right) \mathrm{d} x .
\end{aligned}
$$

Furthermore, (2.6) implies that

$$
N^{-\frac{1}{2}} \Delta_{j}^{-1}\left(1-\frac{\left|\sigma_{j}\right|}{a_{N+1}}\right)^{-\frac{1}{2}} \leq c_{4}
$$

Besides, (2.5) leads to

So using (2.6) again yields that

$$
\left|1-\frac{\left|\sigma_{j}\right|}{a_{N+1}}\right| \geq\left|1-\frac{\sigma_{0}}{a_{N+1}}\right| \geq c_{5} N^{-\frac{2}{3}}
$$

$$
N^{-\frac{1}{2}} \Delta_{j}\left(1-\frac{\left|\sigma_{j}\right|}{a_{N+1}}\right)^{-\frac{1}{2}} \leq c_{6} N^{-1}\left(1-\frac{\left|\sigma_{j}\right|}{a_{N+1}}\right)^{-1} \leq \frac{c_{6}}{c_{5}} N^{-\frac{1}{3}} .
$$

Furthermore $|x| \leq \sqrt{2 N+3}$ for $x \in \Lambda_{j}$, and thus

$$
\int_{\Lambda_{j}} x^{2} \mathrm{e}^{-x^{2}} v^{2}(x) \mathrm{d} x \leq 5 N \int_{\Lambda_{j}} \mathrm{e}^{-x^{2}} v^{2}(x) \mathrm{d} x
$$

By substituting (2.16)-(2.18) into (2.15), we get that

$$
\|v\|_{\omega, N}^{2} \leq 2 c N^{-\frac{1}{6}}\|v\|_{\omega}\|v\|_{1, \omega}+c N^{\frac{2}{3}} \int_{\Lambda} \mathrm{e}^{-x^{2}} v^{2}(x) \mathrm{d} x+c N^{-\frac{1}{3}} \int_{\Lambda} \mathrm{e}^{-x^{2}}\left(\partial_{x} v(x)\right)^{2} \mathrm{~d} x
$$

where $c=\max \left(\frac{c_{1} c_{3}}{2}, \frac{c_{1} c_{2} c_{6}}{c_{5}}, c_{1} c_{2} c_{4}+\frac{5 c_{1} c_{2} c_{6}}{c_{5}}\right)$. The proof is complete.

Theorem 2.1. For any $v \in H_{\omega}^{r}(\Lambda), r \geq 1$ and $0 \leq \mu \leq r$,

$$
\left\|v-I_{N} v\right\|_{\mu, \omega} \leq c N^{\frac{1}{3}+\frac{\mu-r}{2}}\|v\|_{r, \omega} .
$$

Proof. It is proved in [9] that for any $\phi \in \mathcal{P}_{N}$ and $\mu \geq 0$,

$$
\|\phi\|_{\mu, \omega} \leq c N^{\frac{\mu}{2}}\|\phi\|_{\omega}
$$

This fact with Lemma 2.3 lead to that

$$
\begin{aligned}
\left\|P_{N} v-I_{N} v\right\|_{\mu, \omega} & \leq c N^{\frac{\mu}{2}}\left\|P_{N} v-I_{N} v\right\|_{\omega} \leq c N^{\frac{\mu}{2}}\left\|I_{N}\left(v-P_{N} v\right)\right\|_{\omega}=c N^{\frac{\mu}{2}}\left\|v-P_{N} v\right\|_{\omega, N} \\
& \leq c N^{\frac{1}{3}+\frac{\mu}{2}}\left\|v-P_{N}\right\|_{\omega}+c N^{-\frac{1}{6}+\frac{\mu}{2}}\left\|v-P_{N} v\right\|_{1, \omega} .
\end{aligned}
$$


Finally by Lemma 2.1,

$$
\left\|v-I_{N} v\right\|_{\mu, \omega} \leq\left\|v-P_{N} v\right\|_{\mu, \omega}+\left\|I_{N} v-P_{N} v\right\|_{\mu, \omega} \leq c N^{\frac{1}{3}+\frac{\mu-r}{2}}\|v\|_{r, \omega} .
$$

Theorem 2.2. For any $v \in H_{\omega}^{1}(\Lambda)$ and $r \geq 1$,

$$
\left\|\left(v-I_{N} v\right) \mathrm{e}^{-\frac{1}{2} x^{2}}\right\|_{\infty} \leq c N^{\frac{7}{12}-\frac{r}{2}}\|v\|_{r, \omega}
$$

Proof. By (2.1) and Theorem 2.1, for any $x \in \Lambda$,

$$
\begin{aligned}
\left|v(x)-I_{N} v(x)\right| & \leq c \mathrm{e}^{\frac{1}{2} x^{2}}\left\|v-I_{N} v\right\|_{\omega}^{\frac{1}{2}}\left\|v-I_{N} v\right\|_{1, \omega}^{\frac{1}{2}} \\
& \leq c \mathrm{e}^{\frac{1}{2} x^{2}} N^{\frac{7}{12}-\frac{r}{2}}\|v\|_{r, \omega}
\end{aligned}
$$

which implies the desired result.

Theorem 2.3. For any $v \in H_{\omega}^{r}(\Lambda)$ and $r \geq 1$,

$$
\left\|v-I_{N} v\right\|_{\omega, N} \leq c N^{\frac{1}{3}-\frac{r}{2}}\|v\|_{r, \omega}
$$

Proof. By (2.8), for any $\phi \in \mathcal{P}_{N}$,

$$
\left\|v-I_{N} v\right\|_{\omega, N}^{2}=\left(v-I_{N} v, v-\phi\right)_{\omega, N}
$$

whence

$$
\left\|v-I_{N} v\right\|_{\omega, N}=\inf _{\phi \in \mathcal{P}_{N}}\|v-\phi\|_{\omega, N}
$$

By taking $\phi=P_{N} v$ and using Lemma 2.3 and Theorem 2.1, we get that

$$
\left\|v-I_{N} v\right\|_{\omega, N} \leq c N^{\frac{1}{3}}\left\|v-P_{N} v\right\|_{\omega}+c N^{-\frac{1}{6}}\left\|v-P_{N} v\right\|_{1, \omega} \leq c N^{\frac{1}{3}-\frac{r}{2}}\|v\|_{r, \omega} .
$$

We have from (2.8), (2.10) and Theorem 2.1 that for any $v \in H_{\omega}^{r}(\Lambda), \phi \in \mathcal{P}_{N}$ and $r \geq 1$,

$$
\begin{aligned}
\left|(v, \phi)_{\omega}-(v, \phi)_{\omega, N}\right| & =\left|\left(v-I_{N} v, \phi\right)_{\omega}\right| \leq c\left\|v-I_{N} v\right\|_{\omega}\|\phi\|_{\omega} \\
& \leq c N^{\frac{1}{3}-\frac{r}{2}}\|v\|_{r, \omega}\|\phi\|_{\omega} .
\end{aligned}
$$

\section{Applichtion to Burgers equation on the Whole line}

In this section, we consider the Hermite pseudospectral method for the Burgers equation on the whole line. We first change it to an alternative formulation by a similarity transformation, which is suitable for the Hermite approximation. We shall prove the stability and the spectral accuracy of the designed scheme strictly.

Let $\mu>0$ be the kinetic viscosity. $g(y, s)$ and $V_{0}(y, s)$ are the source term and the initial value, respectively. $T$ is a fixed positive number. We consider the following problem

$$
\begin{cases}\partial_{s} V+\frac{1}{2} \partial_{y}\left(V^{2}\right)-\mu \partial_{y}^{2} V=g, & -\infty<y<\infty, 0<s \leq T, \\ V(y, 0)=V_{0}(y), & y \in \tilde{\Lambda} .\end{cases}
$$

In addition, $V$ and $\partial_{y} V$ satisfy certain conditions at the infinity. If we multiply (3.1) by $V(y) \omega(y)$ and sum the result for $\sigma_{j} \in \Lambda_{N}$, then by (2.9) and integration by parts, the last term of the left side of (3.1) becomes

$$
a_{\omega}(V, V)=\int_{\tilde{\Lambda}} \partial_{y} V(y) \partial_{y}(V(y) \omega(y)) \mathrm{d} y, \quad \forall V \in \mathcal{P}_{N} .
$$


It can be checked that

$$
\begin{aligned}
a_{\omega}(V, V) & =\left\|\partial_{y} V\right\|_{L_{\omega}^{2}(\tilde{\Lambda})}^{2}-2 \int_{\tilde{\Lambda}} y V(y) \partial_{y} V(y) \omega(y) \mathrm{d} y \\
& =\left\|\partial_{y} V\right\|_{L_{\omega}^{2}(\tilde{\Lambda})}^{2}+\|V\|_{L_{\omega}^{2}(\tilde{\Lambda})}^{2}-2 \int_{\tilde{\Lambda}} y^{2} V^{2}(y) \omega(y) \mathrm{d} y
\end{aligned}
$$

It is not clear whether or not $a_{\omega}(V, V) \geq 0$. So (3.1) is not suitable for Hermite pseudospectral approximation. To remedy this trouble, we follow Guo [10] to make the similarity transformation

$$
x=\frac{y}{2 \sqrt{\mu(1+s)}}, t=\ln (1+s), U(x, t)=\mathrm{e}^{x^{2}} V\left(2 \sqrt{\mu} x \mathrm{e}^{\frac{t}{2}}, \mathrm{e}^{t}-1\right), f(x, t)=\mathrm{e}^{x^{2}+t} g\left(2 \sqrt{\mu} x \mathrm{e}^{\frac{t}{2}}, \mathrm{e}^{t}-1\right) .
$$

Then (3.1) reads

$$
\begin{cases}\partial_{t} U+\frac{1}{2} U+\frac{1}{2} x \partial_{x} U+\frac{1}{4 \sqrt{\mu}} \mathrm{e}^{x^{2}+\frac{t}{2}} \partial_{x}\left(\mathrm{e}^{-2 x^{2}} U^{2}\right)-\frac{1}{4} \partial_{x}^{2} U=f, & x \in \Lambda, 0<t \leq \ln (1+T) \\ U=U_{0}, & t=0\end{cases}
$$

As in [13], we suppose that $V_{0}$ and $g$ fulfill some conditions such that for certain $\alpha \geq 0$,

$$
\lim _{|y| \rightarrow \infty} \mathrm{e}^{\alpha y^{2}}\left(|V(y, s)|+\left|\partial_{y} V(y, s)\right|\right)=0,0 \leq s \leq T
$$

Then

$$
\lim _{|x| \rightarrow \infty} \mathrm{e}^{\left(4 \alpha \mu \mathrm{e}^{t}-1\right) x^{2}}\left(|U(x, t)|+\left|\partial_{x} U(x, t)\right|\right)=0,0 \leq t \leq \ln (1+T) .
$$

If $\alpha>\frac{1}{8 \mu}$, then for all $t \geq 0,4 \alpha \mu \mathrm{e}^{t}-1>-\frac{1}{2}$. So $U \in H_{\omega}^{1}(\Lambda)$, and we can use the Hermite approximation.

Let $u_{N}$ be the approximation to $U$. The Hermite pseudospectral scheme for (3.2) is to find $u_{N} \in \mathcal{P}_{N}$ for all $t \leq \ln (1+T)$, such that

$$
\begin{cases}\partial_{t} u_{N}+\frac{1}{2} u_{N}+\frac{1}{2} x \partial_{x} u_{N}+\frac{1}{4 \sqrt{\mu}} \mathrm{e}^{x^{2}+\frac{t}{2}} \partial_{x}\left(\mathrm{e}^{-2 x^{2}} u_{N}^{2}\right)-\frac{1}{4} \partial_{x}^{2} u_{N}=I_{N} f, & x \in \Lambda_{N}, 0<t \leq \ln (1+T) \\ u_{N}(0)=u_{N, 0}=I_{N} U_{0} & x \in \Lambda_{N}, t=0\end{cases}
$$

Let

$$
B(v, w, z)=\left(\mathrm{e}^{x^{2}} \partial_{x}\left(\mathrm{e}^{-2 x^{2}} v w\right), z\right)_{\omega, N}
$$

According to (2.10), (3.3) stands for

$$
\begin{cases}\left(\partial_{t} u_{N}(t), \phi\right)_{\omega}+\frac{1}{2}\left(u_{N}(t), \phi\right)_{\omega}+\frac{1}{4 \sqrt{\mu}} \mathrm{e}^{\frac{t}{2}} B\left(u_{N}, u_{N}, \phi\right)+\frac{1}{4}\left(\partial_{x} u_{N}(t), \partial_{x} \phi\right)_{\omega} & =(f(t), \phi)_{N, \omega}, \\ & \forall \phi \in \mathcal{P}_{N}, 0 \leq t \leq \ln (1+T) \\ u_{N}=u_{N, 0}=I_{N} U_{0} & t=0 .\end{cases}
$$

We now consider the stability of (3.3). Since (3.3) is nonlinear, it is not possible to prove the stability in the sense of Courant [4], also see [14]. But it may be stable in the sense of Guo [7,8]. To do this, assume that $f$ and $u_{N, 0}$ have the errors $\tilde{f}$ and $\tilde{u}_{N, 0}$, respectively. They induce the error of $u_{N}$, denoted by $\tilde{u}_{N}$. The errors 
fulfill the following equation

$$
\begin{cases}\left(\partial_{t} \tilde{u}_{N}(t), \phi\right)_{\omega}+\frac{1}{2}\left(\tilde{u}_{N}(t), \phi\right)_{\omega}+\frac{1}{4 \sqrt{\mu}} \mathrm{e}^{\frac{t}{2}} B\left(\tilde{u}_{N}, \tilde{u}_{N}, \phi\right) & +\frac{\mathrm{e}^{\frac{t}{2}}}{2 \sqrt{\mu}} B\left(\tilde{u}_{N}(t), u_{N}(t), \phi\right) \\ +\frac{1}{4}\left(\partial_{x} \tilde{u}_{N}(t), \partial_{x} \phi\right)_{\omega}=(\tilde{f}(t), \phi)_{\omega, N}, & \forall \phi \in \mathcal{P}_{N}, 0 \leq t \leq \ln (1+T) \\ \tilde{u}_{N}(0)=\tilde{u}_{N, 0} & \end{cases}
$$

By taking $\phi=2 \tilde{u}_{N}$ in (3.5), it follows that

$$
\frac{\mathrm{d}}{\mathrm{d} t}\left\|\tilde{u}_{N}(t)\right\|_{\omega}^{2}+\frac{1}{2}\left\|\tilde{u}_{N}(t)\right\|_{1, \omega}^{2}+\frac{\mathrm{e}^{\frac{t}{2}}}{2 \sqrt{\mu}} B\left(\tilde{u}_{N}(t), \tilde{u}_{N}(t), \tilde{u}_{N}(t)\right)+\frac{\mathrm{e}^{\frac{t}{2}}}{\sqrt{\mu}} B\left(\tilde{u}_{N}(t), u_{N}(t), \tilde{u}_{N}(t)\right) \leq 2\|\tilde{f}(t)\|_{\omega, N}^{2} .
$$

In order to estimate the nonlinear term in (3.6), we need the following lemma.

Lemma 3.1. For any $v, w \in H_{\omega}^{1}(\Lambda)$ and $z \in L_{\omega}^{2}(\Lambda)$,

$$
\begin{aligned}
|B(v, w, z)| \leq & c\|z\|_{\omega}^{\frac{1}{2}}\|z\|_{1, \omega}^{\frac{1}{2}}\left(\left\|\partial_{x} v\right\|_{\omega, N}\|w\|_{\omega}^{\frac{1}{2}}\|w\|_{1, \omega}^{\frac{1}{2}}+\left\|\partial_{x} w\right\|_{\omega, N}\|v\|_{\omega}^{\frac{1}{2}}\|v\|_{1, \omega}^{\frac{1}{2}}\right) \\
& +c\|v\|_{\omega}^{\frac{1}{2}}\|v\|_{1, \omega}^{\frac{1}{2}}\|w\|_{\omega}^{\frac{1}{2}}\|w\|_{1, \omega}^{\frac{1}{2}}\|z\|_{\omega, N} .
\end{aligned}
$$

Proof. We have

$$
B(v, w, z)=\sum_{i=1}^{3} B_{i}(v, w, z)
$$

where

By (2.1),

$$
\begin{aligned}
& B_{1}(v, w, z)=\left(\mathrm{e}^{-x^{2}} \partial_{x} v w, z\right)_{\omega, N}, \quad B_{2}(v, w, z)=\left(\mathrm{e}^{-x^{2}} v \partial_{x} w, z\right)_{\omega, N}, \\
& B_{3}(v, w, z)=-2\left(x \mathrm{e}^{-x^{2}} v w, z\right)_{\omega, N} .
\end{aligned}
$$

Similarly

$$
\left|B_{1}(v, w, z)\right| \leq c\left\|\partial_{x} v\right\|_{\omega, N}\|w\|_{\omega}^{\frac{1}{2}}\|w\|_{1, \omega}^{\frac{1}{2}}\|z\|_{\omega}^{\frac{1}{2}}\|z\|_{1, \omega}^{\frac{1}{2}} .
$$

Moreover by (2.1) and (2.10),

$$
\left|B_{2}(v, w, z)\right| \leq c\|v\|_{\omega}^{\frac{1}{2}}\|v\|_{1, \omega}^{\frac{1}{2}}\left\|\partial_{x} w\right\|_{\omega, N}\|z\|_{\omega}^{\frac{1}{2}}\|z\|_{1, \omega}^{\frac{1}{2}} .
$$

$$
\begin{aligned}
\left|B_{3}(v, w, z)\right| & \leq c\|v\|_{\omega}^{\frac{1}{2}}\|v\|_{1, \omega}^{\frac{1}{2}}\|w\|_{\omega}^{\frac{1}{2}}\|w\|_{1, \omega}^{\frac{1}{2}}\|z\|_{\omega, N}\left(\sum_{j=0}^{N} \sigma_{j}^{2} \omega_{j}\right)^{\frac{1}{2}} \\
& \leq c\|v\|_{\omega}^{\frac{1}{2}}\|v\|_{1, \omega}^{\frac{1}{2}}\|w\|_{\omega}^{\frac{1}{2}}\|w\|_{1, \omega}^{\frac{1}{2}}\|z\|_{\omega, N} .
\end{aligned}
$$

The above statements lead to the desired result.

By Lemma 3.1, (2.11) and the Hölder inequality, we obtain that

$$
\begin{aligned}
& \left|B\left(\tilde{u}_{N}(t), \tilde{u}_{N}(t), \tilde{u}_{N}(t)\right)\right| \leq c_{1}(\mu, T)\left\|\tilde{u}_{N}(t)\right\|_{\omega}\left\|\tilde{u}_{N}(t)\right\|_{1, \omega}^{2}, \\
& \left|B\left(\tilde{u}_{N}(t), u_{N}(t), \tilde{u}_{N}(t)\right)\right| \leq c_{2}\left(u_{N}, \mu, T\right)\left\|\tilde{u}_{N}(t)\right\|_{\omega}^{2}+\epsilon\left\|\tilde{u}_{N}(t)\right\|_{1, \omega}^{2}
\end{aligned}
$$

where $c_{1}(\mu, T)$ is some positive constant depending only on $\mu$ and $T$, and

$$
c_{2}\left(u_{N}, \mu, T\right)=\frac{c_{1}(\mu, T)}{\epsilon}\left\|u_{N}\right\|_{L^{\infty}\left(0, \ln (1+T) ; H_{\omega}^{1}(\Lambda)\right)}^{4}, \quad \epsilon>0 .
$$


By substituting (3.7) and (3.8) into (3.6), and integrating it for $t$, we obtain that

$$
\left\|\tilde{u}_{N}(t)\right\|_{\omega}^{2}+\int_{0}^{t}\left(\frac{1}{4}-c_{1}(\mu, T)\left\|\tilde{u}_{N}(\eta)\right\|_{\omega}\right)\left\|\tilde{u}_{N}(\eta)\right\|_{1, \omega}^{2} \mathrm{~d} \eta \leq \rho\left(\tilde{u}_{N, 0}, \tilde{f}, t\right)+c_{2}\left(u_{N}, \mu, T\right) \int_{0}^{T}\left\|\tilde{u}_{N}(\eta)\right\|_{\omega}^{2} \mathrm{~d} \eta
$$

where

$$
\rho\left(\tilde{u}_{N, 0}, \tilde{f}, t\right)=\left\|\tilde{u}_{N, 0}\right\|_{\omega}^{2}+2 \int_{0}^{t}\|\tilde{f}(\eta)\|_{\omega, N}^{2} \mathrm{~d} \eta
$$

Lemma 3.2 (see [10]). Assume that

(i) the constants $b_{1}>0, b_{2} \geq 0, b_{3} \geq 0$ and $d \geq 0$,

(ii) $Z(t)$ and $A(t)$ are non-negative functions of $t$,

(iii) $d \leq \frac{b_{1}^{2}}{b_{2}^{2}} \mathrm{e}^{-b_{3} t_{1}}$ for certain $t_{1}>0$,

(iv) for all $t \leq t_{1}$,

$$
Z(t)+\int_{0}^{t}\left(b_{1}-b_{2} Z^{\frac{1}{2}}(\eta)\right) A(\eta) \mathrm{d} \eta \leq d+b_{3} \int_{0}^{t} Z(\eta) \mathrm{d} \eta
$$

Then for all $t \leq t_{1}, Z(t) \leq d \mathrm{e}^{b_{3} t}$.

Applying Lemma 3.2 to (3.9), we obtain the following result.

Theorem 3.1. Let $\alpha>\frac{1}{8 \mu}$ and $u_{N}$ be the solution of (3.3). If for certain $t_{1}$,

$$
\rho\left(\tilde{u}_{N, 0}, \tilde{f}, t_{1}\right)<\frac{1}{64 c_{1}^{2}(\mu, T)} \mathrm{e}^{-c_{2}\left(u_{N}, \mu, T\right) t_{1}}
$$

then for all $t \leq t_{1}$,

$$
\left\|\tilde{u}_{N}(t)\right\|_{\omega}^{2}+\frac{1}{8} \int_{0}^{t}\left\|\tilde{u}_{N}(\eta)\right\|_{1, \omega}^{2} \mathrm{~d} \eta \leq \rho\left(\tilde{u}_{N, 0}, \tilde{f}, t\right) \mathrm{e}^{c_{2}\left(u_{N}, \mu, T\right) t} .
$$

Theorem 3.1 indicates that the error of the numerical solution is controlled by the errors of the data $u_{N, 0}$ and $f$, provided that the average error $\rho\left(\tilde{u}_{N, 0}, \tilde{f}, t\right)$ does not exceed certain critical value. It means that (3.3) is of generalized stability in the sense of Guo [7,8], and of restricted stability in the sense of Stetter [15].

We next deal with the convergence. Let $U$ be the solution of (3.2), and $U_{N}=P_{N} U$. We derive from (3.2) that

$$
\begin{aligned}
& \left(\partial_{t} U_{N}(t), \phi\right)_{\omega}+\frac{1}{2}\left(U_{N}(t), \phi\right)_{\omega}+\frac{\mathrm{e}^{\frac{t}{2}}}{4 \sqrt{\mu}} B\left(U_{N}(t), U_{N}(t), \phi\right)+\frac{1}{4}\left(\partial_{x} U_{N}(t), \partial_{x} \phi\right)_{\omega}+G(t, \phi)=(f(t), \phi)_{\omega, N} \\
& \forall \phi \in \mathcal{P}_{N}, 0<t \leq \ln (1+T)
\end{aligned}
$$

where

$$
G(t, \phi)=\sum_{i=1}^{4} G_{i}(t, \phi)
$$

and

$$
\begin{aligned}
& G_{1}(t, \phi)=\left(\partial_{t} U(t)-\partial_{t} U_{N}(t), \phi\right)_{\omega}, \\
& G_{2}(t, \phi)=\frac{1}{2}\left(U(t)-U_{N}(t), \phi\right)_{\omega}, \\
& G_{3}(t, \phi)=\frac{1}{4 \sqrt{\mu}} \mathrm{e}^{\frac{t}{2}}\left(\left(\mathrm{e}^{x^{2}} \partial_{x}\left(\mathrm{e}^{-2 x^{2}} U^{2}(t)\right), \phi\right)_{\omega}-\left(\mathrm{e}^{x^{2}} \partial_{x}\left(\mathrm{e}^{-2 x^{2}} U_{N}^{2}(t)\right), \phi\right)_{\omega, N}\right), \\
& G_{4}(t, \phi)=(f(t), \phi)_{\omega, N}-(f(t), \phi)_{\omega} .
\end{aligned}
$$


Let $u_{N}$ be the solution of (3.3), and $\tilde{U}_{N}=u_{N}-U_{N}$. By (3.4) and (3.10), we deduce that

$$
\begin{aligned}
& \left(\partial_{t} \tilde{U}_{N}(t), \phi\right)_{\omega}+\frac{1}{2}\left(\tilde{U}_{N}(t), \phi\right)_{\omega}+\frac{\mathrm{e}^{\frac{t}{2}}}{4 \sqrt{\mu}} B\left(\tilde{U}_{N}(t), \tilde{U}_{N}(t), \phi\right)+\frac{\mathrm{e}^{\frac{t}{2}}}{2 \sqrt{\mu}} B\left(\tilde{U}_{N}(t), U_{N}(t), \phi\right) \\
& +\frac{1}{4}\left(\partial_{x} \tilde{U}_{N}(t), \partial_{x} \phi\right)_{\omega}=G(t, \phi)_{\omega}, \quad \forall \phi \in \mathcal{P}_{N}, 0<t \leq \ln (1+T) .
\end{aligned}
$$

In addition, $\tilde{U}_{N}(0)=0$. Comparing with (3.11) with (3.5), we can derive a result similar to that of Theorem 3.1. But $u_{N}, \tilde{u}_{N, 0}$ and $\tilde{f}$ are now replaced by $U_{N}, \tilde{U}_{N}(0)$ and $G(t, \phi)$, respectively. Therefore we only have to estimate the term $\left|G\left(t, \tilde{U}_{N}(t)\right)\right|$. We first have from Lemma 2.1 that

$$
\begin{aligned}
& \left|G_{1}\left(t, \tilde{U}_{N}(t)\right)\right| \leq c N^{-\frac{r}{2}}\left\|\partial_{t} U(t)\right\|_{r, \omega}\left\|\tilde{U}_{N}(t)\right\|_{\omega} \\
& \left|G_{2}\left(t, \tilde{U}_{N}(t)\right)\right| \leq c N^{-\frac{r}{2}}\|U(t)\|_{r, \omega}\left\|\tilde{U}_{N}(t)\right\|_{\omega}
\end{aligned}
$$

Next

where

$$
4 \sqrt{\mu} \mathrm{e}^{-\frac{t}{2}} G_{3}\left(t, \tilde{U}_{N}(t)\right)=A_{1}\left(t, \tilde{U}_{N}(t)\right)+A_{2}\left(t, \tilde{U}_{N}(t)\right)
$$

$$
\begin{aligned}
A_{1}\left(t, \tilde{U}_{N}(t)\right) & =\left(\mathrm{e}^{x^{2}} \partial_{x}\left(\mathrm{e}^{-2 x^{2}} U^{2}(t)\right), \tilde{U}_{N}(t)\right)_{\omega}-\left(\mathrm{e}^{x^{2}} \partial_{x}\left(\mathrm{e}^{-2 x^{2}} U_{N}^{2}(t)\right), \tilde{U}_{N}(t)\right)_{\omega} \\
& =-\left(\mathrm{e}^{-x^{2}}\left(U^{2}(t)-U_{N}^{2}(t)\right), \partial_{x} \tilde{U}_{N}(t)\right)_{\omega}, \\
A_{2}\left(t, \tilde{U}_{N}(t)\right) & =\left(\mathrm{e}^{x^{2}} \partial_{x}\left(\mathrm{e}^{-2 x^{2}} U_{N}^{2}(t)\right), \tilde{U}_{N}(t)\right)_{\omega}-\left(\mathrm{e}^{x^{2}} \partial_{x}\left(\mathrm{e}^{-2 x^{2}} U_{N}^{2}(t)\right), \tilde{U}_{N}(t)\right)_{\omega, N} .
\end{aligned}
$$

By virtue of (2.1) and Lemma 2.1,

$$
\begin{aligned}
\left|A_{1}\left(t, \tilde{U}_{N}(t)\right)\right| & \leq c\left\|U(t)+U_{N}(t)\right\|_{\omega}^{\frac{1}{2}}\left\|U(t)+U_{N}(t)\right\|_{1, \omega}^{\frac{1}{2}}\left\|U(t)-U_{N}(t)\right\|_{\omega}^{\frac{1}{2}}\left\|U(t)-U_{N}(t)\right\|_{1, \omega}^{\frac{1}{2}}\left\|\tilde{U}_{N}(t)\right\|_{1, \omega} \\
& \leq \frac{1}{16}\left\|\tilde{U}_{N}(t)\right\|_{1, \omega}^{2}+c N^{\frac{1}{2}-r}\left\|U_{N}(t)\right\|_{r, \omega}^{4} .
\end{aligned}
$$

Furthermore

$$
A_{2}\left(t, \tilde{U}_{N}(t)\right)=D_{1}\left(t, \tilde{U}_{N}(t)\right)+D_{2}\left(t, \tilde{U}_{N}(t)\right)
$$

where

$$
\begin{aligned}
& D_{1}\left(t, \tilde{U}_{N}(t)\right)=2\left(\mathrm{e}^{-x^{2}} U_{N}(t) \partial_{x} U_{N}(t), \tilde{U}_{N}(t)\right)_{\omega}-\left(\mathrm{e}^{-x^{2}} U_{N}(t) \partial_{x} U_{N}(t), \tilde{U}_{N}(t)\right)_{\omega, N}, \\
& D_{2}\left(t, \tilde{U}_{N}(t)\right)=-2\left(x \mathrm{e}^{-x^{2}} U_{N}^{2}(t), \tilde{U}_{N}(t)\right)_{\omega}+2\left(x \mathrm{e}^{-x^{2}} U_{N}^{2}(t), \tilde{U}_{N}(t)\right)_{\omega, N}
\end{aligned}
$$

Thanks to (2.20),

We have

$$
\left|D_{1}\left(t, \tilde{U}_{N}(t), \phi\right)\right| \leq c N^{\frac{1}{3}-\frac{r}{2}}\left\|\mathrm{e}^{-x^{2}} U_{N}(t) \partial_{x} U_{N}(t)\right\|_{r, \omega}\left\|\tilde{U}_{N}(t)\right\|_{\omega} .
$$

$$
\partial_{x}^{r}\left(\mathrm{e}^{-x^{2}} U_{N}(t) \partial_{x} U_{N}(t)\right)=\mathrm{e}^{-x^{2}}\left(\partial_{x}^{r} U_{N} \partial_{x} U_{N}+\partial_{x}^{r+1} U_{N} U_{N}\right)+p_{r}(x) \mathrm{e}^{-x^{2}} U_{N} \partial_{x} U_{N}+\cdots
$$

where $p_{r}(x)$ is polynomial of degree $r$. By virtue of (2.1), (2.2) and Lemma 2.1,

$$
\left|D_{1}\left(t, \tilde{U}_{N}(t)\right)\right| \leq c N^{\frac{5}{3}-r}\|U(t)\|_{r, \omega}^{4}+c\left\|\tilde{U}_{N}(t)\right\|_{\omega}^{2} .
$$

We can estimate $D_{2}\left(t, \tilde{U}_{N}(t)\right)$ similarly. Besides, $(2.20)$ implies that

$$
\left|G_{4}\left(t, \tilde{U}_{N}(t)\right)\right| \leq c N^{\frac{2}{3}-r}\|f\|_{r, \omega}^{2}+\|\phi\|_{\omega}^{2} .
$$


TABLE 1. The errors $E_{N}^{(1)}(1)$.

\begin{tabular}{|c|c|c|c|}
\hline$\tau$ & $N=8$ & $N=16$ & $N=32$ \\
\hline 0.01 & $1.389 \mathrm{E}-05$ & $1.389 \mathrm{E}-05$ & $1.389 \mathrm{E}-05$ \\
\hline 0.001 & $1.381 \mathrm{E}-06$ & $1.381 \mathrm{E}-06$ & $1.381 \mathrm{E}-06$ \\
\hline 0.000001 & $1.397 \mathrm{E}-08$ & $1.419 \mathrm{E}-10$ & $2.838 \mathrm{E}-12$ \\
\hline
\end{tabular}

TABLE 2. The errors $\tilde{E}_{N}^{(1)}(1)$.

\begin{tabular}{|c|c|c|c|}
\hline$\tau$ & $N=8$ & $N=16$ & $N=32$ \\
\hline 0.01 & $2.396 \mathrm{E}-03$ & $2.396 \mathrm{E}-03$ & $2.396 \mathrm{E}-03$ \\
\hline 0.001 & $2.382 \mathrm{E}-04$ & $2.382 \mathrm{E}-04$ & $2.382 \mathrm{E}-04$ \\
\hline 0.000001 & $2.409 \mathrm{E}-06$ & $2.447 \mathrm{E}-08$ & $4.895 \mathrm{E}-10$ \\
\hline
\end{tabular}

Therefore

$$
\left|G\left(t, \tilde{U}_{N}(t)\right)\right| \leq \frac{1}{8}\left\|\tilde{U}_{N}(t)\right\|_{1, \omega}^{2}+c\left\|\tilde{U}_{N}(t)\right\|_{\omega}^{2}+c(\mu, T) N^{-r}\left(\|U(t)\|_{r+\frac{5}{3}, \omega}^{4}+\left\|\partial_{t} U(t)\right\|_{r, \omega}^{2}+\|f(t)\|_{r+\frac{3}{2}, \omega}^{2}\right)
$$

Obviously, the last term in the above inequality tends to zero as $N$ goes to the infinity. Finally we obtain the following result.

Theorem 3.2. If $\alpha>\frac{1}{8 \mu}, r \geq 0, U \in L^{2}\left(0, \ln (1+T) ; H_{\omega}^{r+\frac{5}{3}}(\Lambda)\right) \cap H^{1}\left(0, \ln (1+T) ; H_{\omega}^{r}(\Lambda)\right)$ and $f \in$ $L^{2}\left(0, \ln (1+T) ; H_{\omega}^{r+\frac{2}{3}}(\Lambda)\right)$, then for all $t \leq \ln (1+T)$,

$$
\left\|u_{N}(t)-U(t)\right\|_{\omega}^{2}+\int_{0}^{t}\left\|u_{N}(\eta)-U(\eta)\right\|_{1, \omega}^{2} \mathrm{~d} \eta \leq c^{*} N^{-r}
$$

where $c^{*}$ is a positive constant depending only on $\mu, T$ and the norms of $U$ and $f$ in the mentioned spaces.

Remark 3.1. In the proof of Theorem 3.1 and Theorem 3.2, we require that for $U \in H_{\omega}^{1}(\Lambda)$ and so $\mathrm{e}^{-\frac{x^{2}}{2}}\left(|U(x, t)|+\left|\partial_{x} U(x, t)\right|\right) \rightarrow 0$ as $|x| \rightarrow \infty$. A sufficient condition for it is that for certain $\alpha>\frac{1}{8 \mu}$, $\mathrm{e}^{\alpha y^{2}}\left(|V(y, s)|+\left|\partial_{y} V(y, s)\right|\right) \rightarrow 0$, as $|y| \rightarrow \infty$, see [10]. It means that $V(y, s)$ should decay fast enough. It agrees the experience in actual computations as described by Funaro and Kavian [6].

Remark 3.2. In this paper, we use the variable transformation and so obtain the error estimations. In fact, a similar transformation was used in actual computations by Funaro and Kavian [6]. This trick can be generalized to other problems such as the two-dimensional heat equation and the Navier-Stokes equations.

\section{Numerical RESUlts}

We now present some numerical results. Take the following test function

$$
U(x, t)=\operatorname{sech}^{2}(a x-b t-c) .
$$

We use (3.3) to solve (3.2) with $a=0.3, b=0.5, c=-3$ and $\mu=1$. In actual computation, we use standard fourth order Runge-Kutta method in time $t$ with the step $\tau$. Let $E_{N}^{(1)}(t)=\left\|U(t)-u_{N}(t)\right\|_{\omega, N}$ and $\tilde{E}_{N}^{(1)}(t)=$ $\left\|\frac{U(t)-u_{N}(t)}{U(t)}\right\|_{\omega, N}$ be the errors of numerical solution $u_{N}$. Tables 1 and 2 show the errors $E_{N}^{(1)}(t)$ and $\tilde{E}_{N}^{(1)}(t)$ for $t=1$ and various values of $N$ and $\tau$. They indicate the high accuracy and the convergence of the this 
TABLE 3. The errors $E_{N}^{(1)}(t)$ and $\tilde{E}_{N}^{(1)}(t)$.

\begin{tabular}{|c|c|c|}
\hline$t$ & $E^{(1)}(t)$ & $\tilde{E}^{(1)}(t)$ \\
\hline 1.0 & $1.381 \mathrm{E}-06$ & $2.382 \mathrm{E}-04$ \\
\hline 2.0 & $1.249 \mathrm{E}-06$ & $5.848 \mathrm{E}-04$ \\
\hline 3.0 & $8.869 \mathrm{E}-07$ & $1.127 \mathrm{E}-03$ \\
\hline 4.0 & $5.812 \mathrm{E}-07$ & $2.008 \mathrm{E}-03$ \\
\hline 5.0 & $3.680 \mathrm{E}-07$ & $3.456 \mathrm{E}-03$ \\
\hline
\end{tabular}

TABLE 4. The errors $E^{(2)}(1)$.

\begin{tabular}{|c|c|c|c|}
\hline$\tau$ & $N=8$ & $N=16$ & $N=32$ \\
\hline 0.01 & $1.189 \mathrm{E}-05$ & $1.187 \mathrm{E}-05$ & $1.186 \mathrm{E}-05$ \\
\hline 0.001 & $5.965 \mathrm{E}-06$ & $5.883 \mathrm{E}-06$ & $5.883 \mathrm{E}-06$ \\
\hline 0.000001 & $6.510 \mathrm{E}-08$ & $2.380 \mathrm{E}-10$ & $8.194 \mathrm{E}-12$ \\
\hline
\end{tabular}

TABLE 5. The errors $\tilde{E}^{(2)}(1)$.

\begin{tabular}{|c|c|c|c|}
\hline$\tau$ & $N=8$ & $N=16$ & $N=32$ \\
\hline 0.01 & $2.051 \mathrm{E}-03$ & $2.047 \mathrm{E}-03$ & $2.045 \mathrm{E}-03$ \\
\hline 0.001 & $6.028 \mathrm{E}-04$ & $3.014 \mathrm{E}-04$ & $6.698 \mathrm{E}-04$ \\
\hline 0.000001 & $1.122 \mathrm{E}-05$ & $4.106 \mathrm{E}-08$ & $1.431 \mathrm{E}-09$ \\
\hline
\end{tabular}

TABLE 6. The errors $E^{(2)}(t)$ and $\tilde{E}^{(2)}(t)$.

\begin{tabular}{|c|c|c|}
\hline$t$ & $E^{(2)}(t)$ & $\tilde{E}^{(2)}(t)$ \\
\hline 1.0 & $5.883 \mathrm{E}-06$ & $6.698 \mathrm{E}-04$ \\
\hline 2.0 & $3.230 \mathrm{E}-06$ & $1.513 \mathrm{E}-03$ \\
\hline 3.0 & $1.508 \mathrm{E}-06$ & $1.918 \mathrm{E}-03$ \\
\hline 4.0 & $7.377 \mathrm{E}-07$ & $2.549 \mathrm{E}-03$ \\
\hline 5.0 & $4.012 \mathrm{E}-07$ & $3.768 \mathrm{E}-03$ \\
\hline
\end{tabular}

method. Moreover the errors $E_{N}^{(1)}(t)$ and $\tilde{E}_{N}^{(1)}(t)$ with $N=32, \tau=0.001$ and various values of $t$ are listed in Table 3 which shows the stability of calculation.

For comparison, we also use the Hermite spectral method in [10] for the same problem. The corresponding scheme is as follows

$$
\left\{\begin{array}{lr}
\left(\partial_{t} u_{N}(t), \phi\right)_{\omega}+\frac{1}{2}\left(u_{N}(t), \phi\right)_{\omega}+\tilde{B}\left(u_{N}(t), u_{N}(t), \phi\right)+ & \frac{1}{4}\left(\partial_{x} u_{N}(t), \partial_{x} \phi\right)_{\omega}=(f(t), \phi)_{\omega}, \\
& \forall \phi \in \mathcal{P}_{N}, 0<t \leq \ln (1+T),
\end{array}\right.
$$

where

$$
\tilde{B}(u, v, \phi)=-\frac{1}{4 \sqrt{\mu}} \mathrm{e}^{\frac{t}{2}}\left(\mathrm{e}^{-x^{2}} u v, \partial_{x} \phi\right)_{\omega} .
$$

In actual computation, the Hermite coefficients are determined by the Hermite quadratures with the $M$ interpolation points, $M \geq N$. Its numerical errors $E^{(2)}(t)$ and $\tilde{E}^{(2)}(t)$ are defined in the same way as for $E_{N}^{(1)}(t)$ and $\tilde{E}_{N}^{(1)}(t)$. The errors with $M=N$ are shown in Tables 4 and 5 . Moreover the errors with $N=32$ and $\tau=0.001$ are listed in Table 6 . Comparing the errors in Tables 1 to 3 with those in Tables 4 to 6 , we find 
TABLE 7. The errors $E_{N}^{(3)}(1)$.

\begin{tabular}{|c|c|c|c|}
\hline$\tau$ & $N=8$ & $N=16$ & $N=32$ \\
\hline 0.01 & $1.026 \mathrm{E}-03$ & $4.997 \mathrm{E}-04$ & $6.058 \mathrm{E}-05$ \\
\hline 0.001 & $1.075 \mathrm{E}-03$ & $4.992 \mathrm{E}-04$ & $6.030 \mathrm{E}-05$ \\
\hline 0.000001 & $1.075 \mathrm{E}-03$ & $4.991 \mathrm{E}-04$ & $6.027 \mathrm{E}-05$ \\
\hline
\end{tabular}

TABLE 8. The errors $\tilde{E}_{N}^{3}(1)$.

\begin{tabular}{|c|c|c|c|}
\hline$\tau$ & $N=8$ & $N=16$ & $N=32$ \\
\hline 0.01 & $3.568 \mathrm{E}-01$ & $2.283 \mathrm{E}-01$ & $2.977 \mathrm{E}-02$ \\
\hline 0.001 & $3.568 \mathrm{E}-01$ & $2.281 \mathrm{E}-01$ & $2.963 \mathrm{E}-02$ \\
\hline 0.000001 & $3.567 \mathrm{E}-01$ & $2.281 \mathrm{E}-01$ & $2.962 \mathrm{E}-02$ \\
\hline
\end{tabular}

that the Hermite pseudospectral method has the accuracy of the same order as the Hermite spectral method. But the former method saves the work, since it avoids the quadratures on the whole line.

Finally we restrict the computation to a finite interval $\left[Y_{0}, Y_{1}\right], Y_{0}=-25.0$ and $Y_{1}=25.0$. The corresponding artificial boundary values are given by

$$
V\left(Y_{0}, t\right)=V\left(Y_{0}, 0\right), \quad V\left(Y_{1}, t\right)=V\left(Y_{1}, 0\right) .
$$

Take the transformation

$$
\left\{\begin{array}{l}
y=\frac{1}{2}\left(Y_{0}+Y_{1}\right)+\frac{1}{2}\left(Y_{1}-Y_{0}\right) z \\
W(z, s)=V(y, s)-\frac{1}{2}(1+z) V_{0}\left(Y_{1}\right)-\frac{1}{2}(1-z) V_{0}\left(Y_{0}\right)
\end{array}\right.
$$

Then problem (3.1) becomes

$$
\begin{cases}\partial_{s} W+\frac{1}{Y_{1}-Y_{0}} \partial_{z}\left(W+\frac{1}{2}(1+z) V_{0}\left(Y_{1}\right)+\frac{1}{2}(1-z) V_{0}\left(Y_{0}\right)\right)^{2}-\frac{4 \mu}{\left(Y_{1}-Y_{0}\right)^{2}} \partial_{z}^{2} W=g, & z \in(-1,1), t \in(0, T), \\ W(1, s)=W(-1, s)=0, & s \in[0, T), \\ W(z, 0)=V_{0}\left(\frac{1}{2}\left(Y_{1}+Y_{0}\right)+\frac{1}{2}\left(Y_{1}-Y_{0}\right) z\right)-\frac{1}{2}(1+z) V_{0}\left(Y_{1}\right)-\frac{1}{2}(1-z) V_{0}\left(Y_{0}\right), & z \in[-1,1] .\end{cases}
$$

Let $L_{l}(z)$ be the Legendre polynomial of degree $l$, and $\Lambda_{N}^{*}=\left\{z_{j} \mid j=1,2, \ldots N-1\right\}$ be the set of the zeros of $L_{N-1}(z)$. The Legendre pseudospectral scheme for (4.2) is to find $W_{N} \in \mathcal{P}_{N}^{0}(-1,1)$, such that

$$
\begin{cases}\partial_{s} W_{N}+\frac{1}{Y_{1}-Y_{0}} \partial_{z}\left(W_{N}+\frac{1}{2}(1+z) V_{0}\left(Y_{1}\right)+\frac{1}{2}(1-z) V_{0}\left(Y_{0}\right)\right)^{2}-\frac{4 \mu}{\left(Y_{1}-Y_{0}\right)^{2}} \partial_{z}^{2} W_{N}=g, & z \in \Lambda_{N}^{*}, t \in(0, T) \\ W_{N}(z, 0)=V_{0}\left(\frac{1}{2}\left(Y_{1}+Y_{0}\right)+\frac{1}{2}\left(Y_{1}-Y_{0}\right) z\right)-\frac{1}{2}(1+z) V_{0}\left(Y_{1}\right)-\frac{1}{2}(1-z) V_{0}\left(Y_{0}\right), & z \in \Lambda_{N}^{*}\end{cases}
$$

Set $V_{N}(z, s)=W_{N}(z, s)+\frac{1}{2}(1+z) V_{0}\left(Y_{1}\right)+\frac{1}{2}(1-z) V_{0}\left(Y_{0}\right)$. The numerical errors $E_{N}^{(3)}(t)=\left\|V(t)-V_{N}(t)\right\|_{N}$ and $\tilde{E}_{N}^{(3)}(t)=\left\|\frac{V(t)-V_{N}(t)}{V(t)}\right\|_{N}$ are presented in Tables 7 to 9 , where $\|v\|_{N}$ is the corresponding Legendre discrete norm. Comparing the results in Tables 1 to 3 with those in Tables 7 to 9, we know that the Hermite pseudospectral method provides better numerical results than the usual method by restricting the computation to a finite interval. In particular, for $N=32$, the points in the Hermite pseudospectral method are sampled over an interval of size $\left|\sigma_{N}\right| \sim \sqrt{2 N}=8$. The size of this interval is still smaller than the truncated interval of size 25. But the Hermite pseudospectral method provides much better results than (4.3). 
TABLE 9. The errors $E_{N}^{(3)}(t)$ and $\tilde{E}_{N}^{(3)}(t)$.

\begin{tabular}{|c|c|c|}
\hline$t$ & $E^{(1)}(t)$ & $\tilde{E}^{(1)}(t)$ \\
\hline 1.0 & $6.030 \mathrm{E}-05$ & $2.963 \mathrm{E}-02$ \\
\hline 2.0 & $3.213 \mathrm{E}-05$ & $2.137 \mathrm{E}-02$ \\
\hline 3.0 & $1.893 \mathrm{E}-05$ & $1.562 \mathrm{E}-02$ \\
\hline 4.0 & $1.229 \mathrm{E}-05$ & $1.126 \mathrm{E}-02$ \\
\hline 5.0 & $9.869 \mathrm{E}-06$ & $1.103 \mathrm{E}-02$ \\
\hline
\end{tabular}

Acknowledgements. The work of the first author is supported by the Chinese State Key project G1999032804.

\section{REFERENCES}

[1] R.A. Adams, Sobolev Spaces. Academic Press, New York (1975).

[2] C. Bernardi and Y. Maday, Spectral methods, in Techniques of Scientific Computing, Part 2, P.G. Ciarlet and J.L. Lions Eds., Elsevier, Amsterdam (1997) 209-486.

[3] O. Coulaud, D. Funaro and O. Kavian, Laguerre spectral approximation of elliptic problems in exterior domains. Comp. Mech. Appl. Mech. Eng. 80 (1990) 451-458.

[4] R. Courant, K.O. Friedrichs and H. Levy, Über die partiellen differezengleichungen der mathematischen physik. Math. Annal. $100(1928) 32-74$.

[5] D. Funaro, Estimates of Laguerre spectral projectors in Sobolev spaces, in Orthogonal Polynomials and Their Applications, C. Brezinski, L. Gori and A. Ronveaux Eds., Scientific Publishing Co. (1991) 263-266.

[6] D. Funaro and O. Kavian, Approximation of some diffusion evolution equations in unbounded domains by Hermite functions. Math. Comp. 57 (1990) 597-619.

[7] B.Y. Guo, A class of difference schemes of two-dimensional viscous fluid flow. TR. SUST (1965). Also see Acta Math. Sinica 17 (1974) 242-258.

[8] B.Y. Guo, Generalized stability of discretization and its applications to numerical solution of nonlinear differential equations. Contemp. Math. 163 (1994) 33-54.

[9] B.Y. Guo, Spectral Methods and Their Applications. World Scientific, Singapore (1998).

[10] B.Y. Guo, Error estimation for Hermite spectral method for nonlinear partial differential equations. Math. Comp. 68 (1999) 1067-1078.

[11] A.L. Levin and D.S. Lubinsky, Christoffel functions, orthogonal polynomials, and Nevaiś conjecture for Freud weights. Constr. Approx. 8 (1992) 461-533.

[12] D.S. Lubinsky and F. Moricz, The weighted $L_{p}$-norm of orthogonal polynomial of Freud weights. J. Approx. Theory 77 (1994) $42-50$.

[13] Y. Maday, B. Pernaud-Thomas and H. Vandeven, Une réhabilitation des méthodes spectrales de type Laguerre. Rech. Aérospat. 6 (1985) 353-379.

[14] R.D. Richitmeyer and K.W. Morton, Finite Difference Methods for Initial Value Problems, 2nd ed., Interscience, New York (1967).

[15] H.J. Stetter, Stability of nonlinear discretization algorithms, in Numerical Solutions of Partial Differential Equations, J. Bramble Ed., Academic Press, New York (1966) 111-123.

[16] G. Szegö, Orthogonal Polynomials. Amer. Math. Soc., New York (1967).

[17] A.F. Timan, Theory of Approximation of Functions of a Real Variable. Pergamon Press, Oxford (1963).

To access this journal online:

www.edpsciences.org 